Communications in Physics, Vol. 24, No. 3S2 (2014), pp. 128-132

DOI:10.15625/0868-3166/24/3S2/5058

\title{
FLUORESCENT PROPERTIES OF CANCEROUS LIVER TISSUE
}

\author{
NGUYEN THI KHANH VAN, NGUYEN DINH HOANG, NGUYEN THANH BINH, \\ NGUYEN CONG THANH \\ Institute of Physics, Vietnam Academy of Science and Technology
}

PHAM KIM BINH

Viet Duc Hospital, Hanoi, Vietnam

E-mail: ntkvan@iop.vast.ac.vn

Received 04 April 2014

Accepted for publication 24 May 2014

\begin{abstract}
Fluorescence spectroscopy is a technology used fruitfully for biomedical diagnostics as well as for therapeutic purposes. The fluorescent analysis method are applied in biomedical diagnostics based on fluorescence of endogenous or exogenous fluorophores. We initially applied fluorescence spectroscopy for the study of human liver cancer (hepatocellular carcinoma) with an exogenous fluorophores, Radachlorin ${ }^{\circledR} 0.35 \%$. The main aim of this study is to determine the spectral variation between normal and malignant liver tissues in 2 cases: the samples exposed and non-exposed to Radachlorin. The excitation wavelength used for the fluorescence measurements is $405 \mathrm{~nm}$.
\end{abstract}

Keywords: autofluorescence, Radachlorin ${ }^{\circledR}$, hepatocellular carcinoma, liver cancer.

\section{INTRODUCTION}

Liver cancer is the third most common cause of cancer-deaths after lung, stomach cancers. Liver cancer in males occurs more common than in females. Development of early detection methods plays an important role in therapy and has great influence on therapeutic effect.

The exploration of new methods and techniques for the diagnosis of tumors has been developed quickly in the recent years. Optical spectroscopy is one of these methods which have many advantages such as simple, rapid, non-invasive,... The measurement of fluorescence from tissue has become a clinical diagnostic tool, especially to assess tissue malignancy or tissue function. The underlying physical basis is that they are strongly influenced by cellular structure and chemical composition of the tissues [1,2].

Morphological and biochemical changes in a diseased tissue cause the fluorophores inside the tissue to fluoresce differently. Due to its sensitivity to minute variations, fluorescence spectroscopy can provide both qualitative and quantitative biochemical information about the state of the tissue, which may not be obtained by using standard pathology. In the recent past, its diagnostic potential has been tested in different organs of the body, including the mouth, breast, esophagus, and bladder, etc. The fact that the tissue is a complex medium, the intrinsic spectra is

(C)2014 Vietnam Academy of Science and Technology 
substantially modified by the medium before its detection. Both characteristic and statistical features of the spectra can reflect the nature and condition of the tissue. In fluorescence spectroscopy, the fluorophores are active under excitation of the ultraviolet and visible light to be useful identifiers [2-4].

Biological tissue contains numerous endogenous fluorophores such as flavins, nucleotides (NADH), tryptophan, tyrosine, elastin, collagen etc. Tissue autofluorescence can be used to help characterise the tissue components and provide contrast between different types and states of tissue. For example in cancers of epithelial origin there is a greater contribution to the surface autofluorescence from NADH (due to an increased of number of metabolically active cells) and a reduced contribution from collagen (due to thickening of the mucosal layer pushing the collagenrich sub-mucosal layer further away from the tissue surface) $[2,5]$.

Besides autofluorescence due to endogenous fluorophores, a large number of exogenous fluorophores (photosensitizer) have been created and studied as well to be used for biological diagnostics. These fluorophores have been created for many different purposes and applications in the field of biological monitoring such as monitoring cellular function using fluorescent reporter dyes or molecules for various biochemical species.

The aim of this study is to determine the spectral variation between normal and malignant liver tissues in 2 cases: the samples exposed and non-exposed to photosensitizer, Radachlorin ${ }^{\circledR}$ $0.35 \%$.

\section{METHODS AND MATERIALS}

\section{Photosensitizer}

The Radachlorin ${ }^{\circledR}$ was purchased from the RADA-PHARMA group (RADA-PHARMA Co, Ltd., Moscow, Russia), which was stable in solutions at $0 \pm 8^{\circ} \mathrm{C}$ in the dark. Radachlorin ${ }^{\circledR}$ was diluted with distilled water at ratio of 1: 15 . Radachlorin fluoresces from the wavelength of $646 \mathrm{~nm}$ to the wavelength of $680 \mathrm{~nm}[6,7]$.

\section{Biological samples}

Diseased human liver (hepatocellular carcinoma) which cut out of a patient was soaked in formaldehyde solution $10 \%$. The liver samples contained both the areas of normal and malignant tissues were cut into thin slices of $0.5 \mathrm{~mm}$. Fig. 1 is an image of the diseased liver cut out of patient.

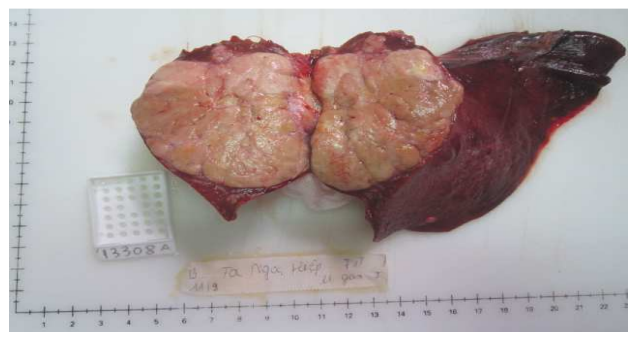

Fig. 1. Hepatocellular carcinoma in human. 
These samples were divided into two groups: Radachlorin exposed group (group 1) and Radachlorin non-exposed group (group 2).

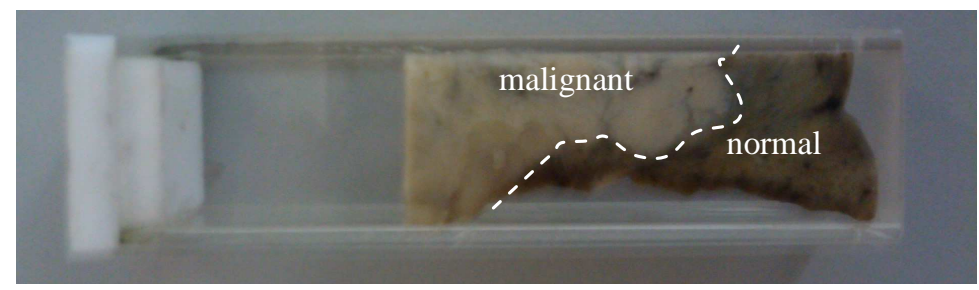

Fig. 2. Hepatocellular carcinoma sample was cut from human liver.

\section{Radachlorin uptake by liver samples}

Biological samples of group 2 were soaked in Radachlorin in 24 hours at room temperature. After a predetermined time, the Radachlorin solution was discarded from the surface of samples: the samples were washed three times with distilled water. Then they were put in a cuvette. Fig. 2 shows a hepatocellular carcinoma sample cut into a thin slice $0.5 \mathrm{~mm}$ and put in the cuvette.

\section{Measurement of tissue samples}

Fluorescence spectra were measured by fluorescence spectrophotometer Varian Cary Eclipse (Australia) with an excitation wavelength at $405 \mathrm{~nm}$ (corresponds to the absorption due to endogenous fluorophore, porphyrin). The cuvette contains the tissue sample is placed in the sample holder in the instrument. The schematic diagram of the instrument is shown in Fig. 3 [8].

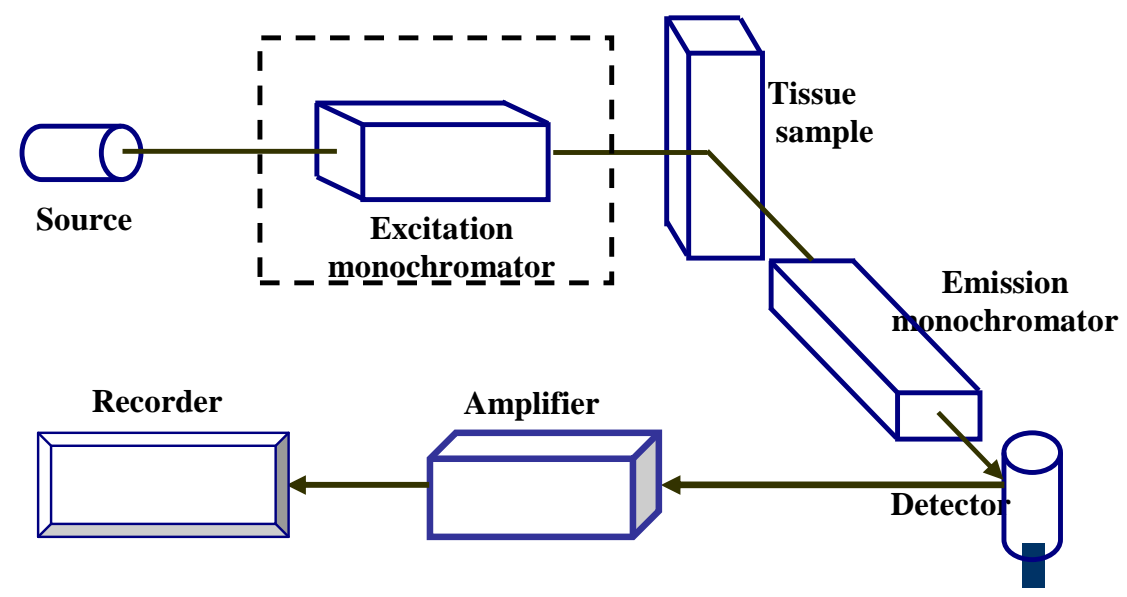

Fig. 3. Schematic diagram of the basic fluorescence spectrophotometer.

Each sample was measured at three different areas: malignant area, normal area and boundary between normal and malignant area. 


\section{RESULTS AND DISCUSSIONS}

The fluorescence emission spectrum of liver tissue samples of group 1 recorded at excitation wavelength of $405 \mathrm{~nm}$ has emission wavelength at $553 \mathrm{~nm}$.

Fig. 4 shows the fluorescence spectra of group 1. It is observed that the shape of the emission spectrum of the malignant tissue area is similar to that of the normal and boundary tissue area. However, there is a small difference in the intensity value of the spectrum. This value increases from the normal area to the malignant area. The intensity value at the malignant area is highest

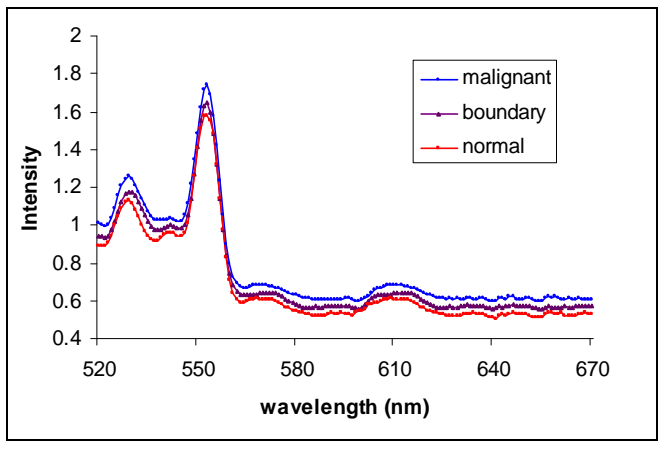

Fig. 4. Fluorescence spectra of Radachlorin nonexposed tissue samples (group 1) excited at wavelength of $405 \mathrm{~nm}$.

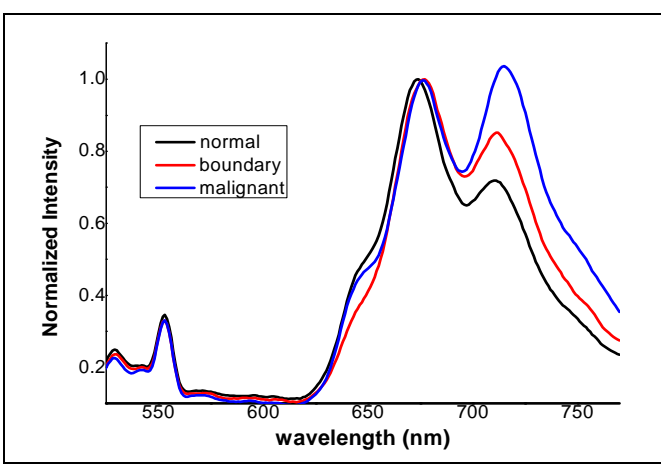

Fig. 5. Normalized fluorescence spectra of Radachlorin exposed tissue samples (group 2) excited at wavelength of $405 \mathrm{~nm}$.

Fig. 5 shows the difference in the intensity value between the normal and the malignant tissue area becomes clearer when the tissue sample is exposed to photosensitizer (group 2). When the tissue sample is exposed to Radachlorin solution and excited at a wavelength of $405 \mathrm{~nm}$, the fluorescence spectra have 2 peaks at $677 \mathrm{~nm}$ and $715 \mathrm{~nm}$. In this case, we can see that the difference of the height of spectrum peaks of the areas is relatively clear. The height of spectrum peak gradually decreases from the malignant area to the normal area.

\section{CONCLUSIONS}

The fluorescence emission spectroscopic technique was employed for the qualitative spectral analysis of hepatocellular carcinoma in human liver. The fluorescence measurements were carried out at $405 \mathrm{~nm}$ excitation wavelength, which corresponds to the absorption due to endogenous fluorophore, on all the two sample groups. The results show that the intensity value between the malignant tissue area and the normal tissue area is different. This value gradually decreases from the malignant area to the normal area. The difference of value intensity in photosensitizer exposed case is clearer than that in photosensitizer non-exposed case. This result is very helpful in diagnosing cancer.

However, this is only the initial results. In the next time, we will carry out more experiments to affirm that it is possible to use diagnostic techniques based on fluorescence spectroscopy to early diagnose cancer. 


\section{REFERENCES}

[1] Mohan, Surapaneni Krishna. et al., Life Sciences \& Medicine Research 2010 (2010) 1.

[2] Anita H. Gharekhan et al., Characterization of Cancer and normal tissue fluorescence Through Wavelet Transform and Singular Value Decomposition, Proc SPIE 03/2008; doi:10.1117/12.768912

[3] Pieter Albert Arthuur De Beule, "Development of Multidimensional Fluorescence Instrumentation for Biomedical Applications, Imperial College London. 2007.

[4] Paul J Tadrous, Jan Siegel, Paul MW French, Sami Shousha, El-Nasir Lalani and Gordon WH Stamp. Journal of Pathology 199 (2003) 309-317.

[5] Lennart Enerback and Ove Lundvall, Virchows Arch. Abt. A Path. Anat. 350, Springer Verlag, pp. 293-302, 1970.

[6] Su-mi Bae, Yong-Wook Kim, Joon-Mo lee, Sung-Eun Namkoong, Sei-Jun Han, Jong-Ki Kim, Chang-Hee Lee, Heung-Jae Chun, Hyun-Sun Jin, and Woong-Shick Ahn, Cancer Research and Treatment 36(6) (2004) 389-394.

[7] Fanklin Vargasand, Yrene Díaz, Viatcheslav Yartsev, Arístides Marcano and Alexander Lappa, Ciencia 12(1) (2004).

[8] Cary Eclipse Fluorescence Spectrophotometer. Available from: http://www.chem.agilent.com/Library/brochures/ Cary-Eclipse_FLR-brochure.pdf 